\title{
Iron Carboxylate Oxygen-Centered-Triangle Complexes Detected During Electrospray Use of Organic Acid Modifiers with a Comment on the Finnigan TSQ-700 Electrospray Inlet System
}

\author{
Carl F. ljames
}

Section on Analytical Biochemistry, Laboratory of Clinical Science, National Institute of Mental Health, Bethesda, Maryland, USA

Robert C. Dutky and Henry M. Fales

Chemical Structure Section, Laboratory of Biophysical Chemistry, National Heart, Lung, and Blood Institute, Bethesda, Maryland, USA

Use of infusion methods rather than high-performance liquid chromatography allowed us to confirm the observation that solutions of propionic acid-isopropanol restore sensitivity lost due to trifluoroacetic acid in electrospray mass spectra of basic substances, particularly peptides. In this work, when propionic acid-isopropanol was used, we detected an abundant ion with $m / z 622$ that shifted to $m / z 538$ when we substituted acetic acid-methanol for the propionic acid-isopropanol. Via accurate mass measurement and tandem mass spectrometry the origin of the ion was identified as the complex $\mathrm{Fe}_{3} \mathrm{O}_{2}\left(\mathrm{O}_{2} \mathrm{CR}\right)_{6}(\mathrm{~L})_{0-3}$, where $\mathrm{L}$ is one of several ligands from solvent or water. The grounding arrangement of the Finnigan TSQ-700 electrospray source produces electrolytic currents that may accentuate the abundance of this complex and specifically produces observable gas bubbles that adversely affect the spray stability. (J Am Soc Mass Spectrom 1995, 6, 1226-1231)

$\mathrm{T}$ The deleterious effect of small amounts of hydrochloric, sulfuric, phosphoric, and especially trifluoroacetic acid (TFA) on electrospray mass spectra of basic compounds such as peptides is well known [1], although its cause is still a matter for some speculation. With TFA the effect is particularly unfortunate because many of the most useful high-performance liquid chromatography (HPLC) systems rely on this additive. Apffel et al. [2] have recently reported that addition of a solution of 75:25 propionic acid-isopropanol added one third by volume postcolumn via a mixing tee at a total flow rate of $300 \mu \mathrm{L} / \mathrm{min}$ before the pneumatic nebulizer nozzle, increases by fifteen- to thirtyfold the signal of basic compounds (peptides and alkaloids) whose signals had been drastically reduced by $0.1-0.2 \%$ TFA. The reported spectra in fact show even greater signal enhancements.

We attempted to extend the technique to simple infusion analysis of peptides at low flow rates (1-3 $\mu \mathrm{L} / \mathrm{min}$ ) by addition of propionic acid-isopropanol to peptides prior to analysis. After our triple quadrupole instrument was tuned with a standard mixture of 5- $\mu \mathrm{M}$ equine apomyoglobin and $20-\mu \mathrm{M}$ L-methionyl-

Address reprint requests to Dr. Henry M. Fales, National Institutes of Health, Building 10, Room 7N-318, 10 Center Drive MSC 1676 Bethesda, MD 20892-1676. arginyl-phenylalanyl-alanine hydroacetate (MRFA) in $50 \%$ aqueous methanol that contained $0.5 \%$ acetic acid (Figure 1a), we observed the not unexpected loss of maximum apomyoglobin signal by a factor of 19 and MRFA by 13 (note signal strengths on right of figure) after addition of TFA to a final concentration of $0.1 \%$ (Figure 1b). The most abundant charge state of apomyoglobin shifted from +18 to +14 and some increased tailing was noticed due to adduct formation. Although proteins were specifically omitted from compounds claimed to be aided by this mixture, we find that addition of $25 \% \mathrm{v} / \mathrm{v}$ of the $75: 25$ propionic acid-isopropanol mixture reduced the signal loss of apomyoglobin to a factor of 14 (Figure 1c), even though the envelope of charge flattened and the degree of adduct tailing became severe. However, the effect of the propionic acid-isopropanol mixture on the MRFA component of the tuning solution was impressive; it actually increased its intensity by a factor of 1.8 over the original solution.

Although these experiments indeed confirmed the value of the propionic acid-isopropanol mixture, our attention was drawn to the abundant ion with $\mathrm{m} / \mathrm{z}$ 622 (Figure 1c), which can dominate the spectrum, dependent on analyte concentration. Closer inspection (Figure 1c inset) revealed a complex isotopic pattern that suggested the presence of metal atoms. We report 

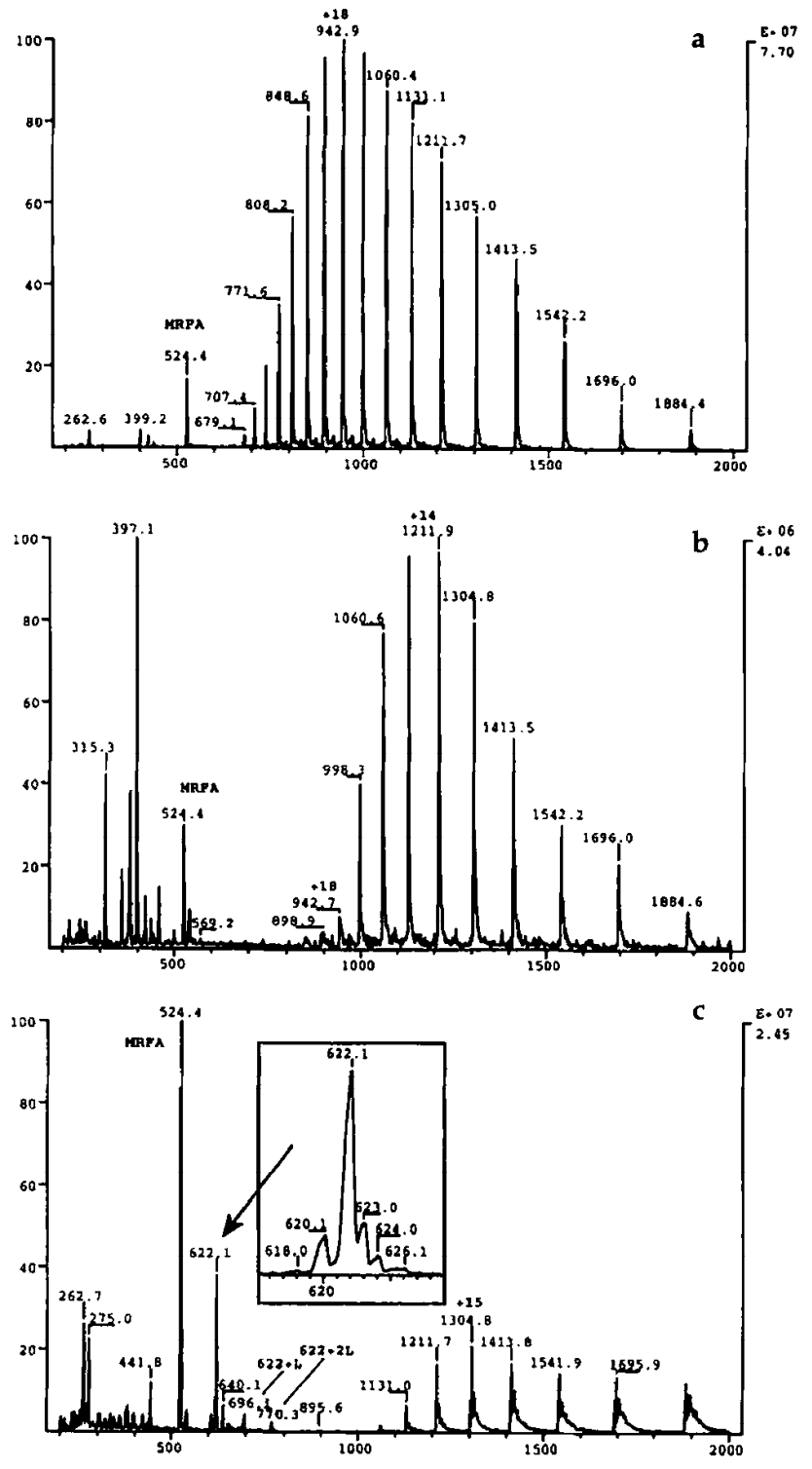

Figure 1. (a) Electrospray spectrum (Finnigan TSQ-700) of 5- $\mu \mathrm{M}$ equine apomyoglobin and $20-\mu \mathrm{M}$ L-methionyl-arginyl-phenylalanyl-alanine hydroacetate (MRFA) in $50 \%(v / v)$ aqueous methanol that contained $0.5 \%$ acetic acid. Note signal strengths on the right of the figure. (b) Same solution as (a) after addition of trifluoroacetic acid to a final concentration of $0.1 \%(v / v)$. (c) same solution as (b) after addition of $25 \%(\mathrm{v} / \mathrm{v})$ propionic acid-isopropanol. The inset shows the iron complex ion with $m / z$ 622. Note the propionic acid ligands with $m / z \quad 696.1$ and 770.3 .

analysis of this peak, its likely source, and further experiments with the propionic acid-isopropanol mixture.

\section{Experimental}

Mass spectra were obtained by using two spectrometers. The first was a Finnigan TSQ-700 triple quadrupole mass spectrometer (Finnigan-MAT, San Jose, CA) equipped with a Finnigan electrospray source that used an rf-only octapole ion guide. Solutions were infused via a motor driven $100-\mu \mathrm{L}$ glass syringe with a stainless steel needle that was coupled to two sequential lengths of $100-\mu \mathrm{m}-\mathrm{i}$.d. fused silica capillary tubing via a Teflon coupling at the syringe needle and an intermediate low dead volume stainless steel union between the two lengths of fused silica capillary. The second length of capillary then enters the spectrometer source inside a 26-gauge stainless steel needle that is maintained at +2300 to $+4500 \mathrm{~V}$. The fused silica capillary ends approximately flush with the surrounding 26-gauge needle, and the expelled solution is considered to contact the metallic needle briefly, which enables electrostatic charging of the solution (Finnigan instrument manual).

The motor driven syringe is electrically insulated, but for safety reasons, the intermediate stainless steel union is electrically bonded to earth ground. We have found this grounding to have a significant analytical effect.

Solutions of peptides and proteins were admitted at a flow rate of $1-3 \mu \mathrm{L} / \mathrm{min}$. Mass spectra were scanned from $m / z 10$ to 2000 in $3 \mathrm{~s}$, data were collected in the profile mode, and spectra were averaged for at least 3 min. Spectra also were acquired by decreasing the potential of the "tube lens" to cause less dissociation of weakly bound adduct ions in the 1-torr pressure region in front of the skimmer. Tandem mass spectrometry analysis was effected by collision with argon gas (usually at 1-3 millitorr) in the second (rf-only) octapole.

Stock solutions of $0.1-\mathrm{M} \mathrm{FeCl}_{3}$ (Chem Services, Inc., West Chester, PA) in propionic or acetic acid were prepared to confirm the nature of the ion with $\mathrm{m} / \mathrm{z}$ 622. Peptides were obtained from Peninsula Laboratories, Inc. (Belmont, CA) and were dissolved in 50\% aqueous methanol.

The second instrument [3] was an FTMS-2000 (Extrel-FTMS, Millipore Corp., Madison, WI) Fourier transform mass spectrometer (FTMS) equipped with a 3-T superconducting magnet and an electrospray ionization source (Analytica of Branford, Branford, CT). To determine the structure of the $m / z 622$ ion, a $5-\mu \mathrm{M}$ solution of gramicidin-S in a mixture of 1:1:1 (propionic acid-isopropanol:methanol:0.2\% aqueous TFA) was electrosprayed from the stainless steel needle assembly at a flow rate of $1.5 \mu \mathrm{L} / \mathrm{min}$, and ions were accumulated for $30-45 \mathrm{~s}$ before FTMS excitationdetection at a resolution of 90,000 at $\mathrm{m} / \mathrm{z} 622$.

\section{Results}

Inspection of the isotope pattern in the quadrupole spectra (Figure 1c, inset) suggested that the ion with $m / z 622$ contained three atoms of iron. To confirm this, four spectra were acquired with the FTMS instrument (whose inlet system exhibited the same ion) to yield an average value for the most abundant ion of $621.9722 \pm 0.0046( \pm 7.2 \mathrm{ppm})$. This differs from $\mathrm{C}_{18} \mathrm{H}_{30} \mathrm{O}_{13} \mathrm{Fe}_{3}$ by only $1.1 \mathrm{ppm}$. The presence of propi- 


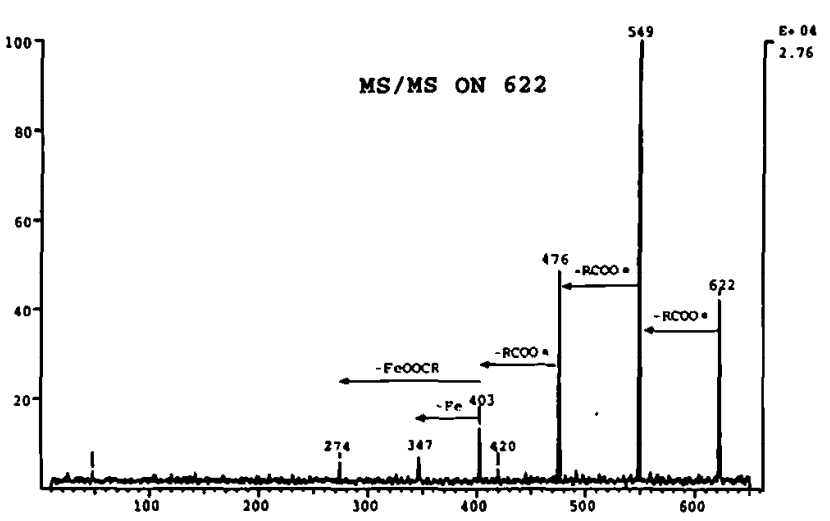

Figure 2. Tandem mass spectrometry of $m / \approx 622$ with argon as the collision gas at 3.0 mtorr with an offset voltage of $10.1 \mathrm{~V}$.

onoxy groups in the cluster is clear from its fragmentation pattern in the triple stage quadrupole that used tandem mass spectrometry with argon as the collision gas (Figure 2). Product ions that corresponded to loss of one, two, and three 73-u propionoxy groups, then loss of an atom of iron, and then another propionoxy group were observed, although the actual losses were not necessarily in that order. Six propionoxy groups account for 12 of the oxygen atoms, whereas the presence of the extra oxygen suggests the ion with $m / z$ 622 is derived from a "basic iron carboxylate" [4] as shown in Structure 1.

These basic iron carboxylates, also known as oxygen-centered triangles $[4,5]$ because their three metal atoms and lone oxygen form a nearly planar triangular unit, have the general formula $\mathrm{Fe}_{3} \mathrm{O}\left(\mathrm{O}_{2} \mathrm{CR}\right)_{6}(\mathrm{~L})_{3}$, where $\mathrm{L}$ is a ligand such as water or pyridine. They are obtained as neutral compounds by heating simple iron salt solutions such as ferric chloride and aqueous carboxylic acids in the presence of air [6] and they are thought to have relevance to the ferritin iron storage protein [7]. Note that only two of the three propionic acid ligands ( $m / z 696$ and 770 ) are observed along with the ion with $m / z 622$ (Figure 1c).

These basic carboxylates often are described in their acetate forms $[4,5]$, so it was not a surprise to find an

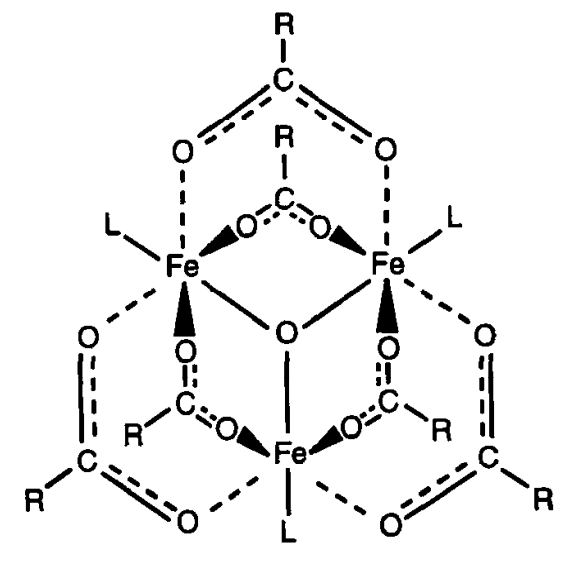

Structure 1 entirely analogous cluster of peaks with $m / z 538$, again with weak ligand signals at $m / z 598$ and 658, when acetic acid was substituted for propionic acid in the preceding experiment. In fact, after inspection of several older spectra, we realized that this set of ions often attended our use of acetic acid solutions. Infusion of a simple 1:1 aqueous methanol solution that contained $20 \%$ acetic acid indeed revealed this ion, but its intensity appeared to vary dependent on the previous history of the apparatus. The ion most likely is derived from dissolution of iron from the stainless steel needle that surrounds the fused silica capillary, either by direct or subsequent reaction with the carboxylic acid. Blades et al. [8] have proposed that the electrospray current itself produces an electrochemical effect, and their studies of the effects of zinc and stainless steel spray tips have shown the production of $\mathrm{Zn}^{++}$and $\mathrm{Fe}^{++}$ions solvated with dimethylsulfoxide.

As configured by the manufacturer, the inlet system of our Finnigan TSQ-700 spectrometer produces a more conventional electrolytic process that greatly exceeds the true electrospray current. Indeed, the so-called electrospray current displayed on the computer terminal is actually a combination of the true electrospray current plus a conventional electrolysis current that runs back through the solvent-filled fused silica capillary to the grounded stainless steel fitting. When the grounding connection is removed from the stainless steel fitting, virtually no current is observed even in the presence of a strong electrospray ion signal because the first small division on the software current display represents $0.32 \mu \mathrm{A}$. This greatly exceeds the true electrospray current. With the ground connection in place as recommended by Finnigan, solutions of moderate conductivity supply an erratic ion signal and spray due to formation of hydrogen gas bubbles by electrolysis of the solution at the grounded fitting. These bubbles are entrained by the fused silica capillary that leads to the electrospray nozzle where they disrupt the spray. With an "electrospray current" of 50 $\mu \mathrm{A}$ indicated on the software display, we have observed bubbles of approximately $0.25-\mu \mathrm{L}$ volume (30 $\mathrm{mm}$ in length) that pass through the $100-\mu \mathrm{m}$-i.d. silica capillary every $30-60 \mathrm{~s}$. By computing the electrolytic effect on water of a 50- $\mu \mathrm{A}$ current, we would expect to produce a volume of $0.25 \mu \mathrm{L}$ of hydrogen gas in $38 \mathrm{~s}$; this is essentially what we observed. This problem of electrolytically formed gas bubbles is especially severe when high conductivity solutions such as propionic acid-isopropanol are used if the stainless steel fitting is left grounded. We now routinely operate with the ground connection removed, although this presents a safety concern when the high voltage is on. (Although this arrangement can be tolerated when an insulated infusion syringe is used, attachment of an ungrounded HPLC system appears to present difficulties.) With the ground connection removed, current is observed on the software current display only when a corona discharge is present. At a $15-\mathrm{mm}$ spacing between the 
spray needle and the heated metal acceptance tube, corona discharge starts at about $+6.3 \mathrm{kV}$ with an $0.3-\mu \mathrm{A}$ current and rises to approximately $4 \mu \mathrm{A}$ at 8 $\mathrm{kV}$. These currents are independent of any liquid infusion, and are present even with a completely dry needle. Similar currents have been observed in the negative ion mode of operation.

To confirm the nature of the iron-containing ion, 1-mM solutions of $\mathrm{FeCl}_{3}$ in acetic or propionic acid were prepared with a variety of cosolvents. All solutions provided intense ions as expected at either $m / z$ 538 or 622 dependent on the acid (not shown). However, the actual compound present in solution is considered to be the triply liganded Structure 1 ( $M W=718$ for $\mathrm{R}=\mathrm{CH}_{3}$ and $\mathrm{L}=\mathrm{CH}_{3} \mathrm{COOH}$; $\mathrm{MW}=844$ for $\mathrm{R}=$ $\mathrm{CH}_{3} \mathrm{CH}_{2}$ and $\mathrm{L}=\mathrm{CH}_{3} \mathrm{CH}_{2} \mathrm{COOH}$ ), which suggests that the lower mass ions actually observed in the spectrometer might be the result of collisional processes that occurred prior to mass analysis by the quadrupole. Indeed, as the tube lens-skimmer potential was reduced from 66 to $0 \mathrm{~V}$ at the mid scan mass, ions that contained up to three acetic acid ligands replaced $m / z 538$ (Figure 3a, b, and $c$ ) as expected for its structure. Similar results were obtained with a wide variety of other cosolvents, which included water, acetonitrile, and tetrahydrofuran, all of which adducted in combinations with the carboxylic acid to total three molecules of ligand. The intensity of these adducted ions is affected not only by reduction of the tube lens potential, but also by the presence of auxiliary gas in the spray region. When dry air was admitted by this route, solvent adduction at a given tube lens potential was reduced strongly (compare Figure $3 b$ and $d$ ).

Interestingly, such neutral basic iron carboxylates are found to have iron in a combination of one $\mathrm{Fe}^{++}$ and two $\mathrm{Fe}^{+++}$states and are referred to as mixedvalence complexes [4,5]. Many examples of such complexes with transition elements are known; the metal atoms are in some cases analytically indistinguishable (Mossbauer spectroscopy) with equivalent formal charges of $+2 \frac{2}{3}$, and in other cases are of mixed valences [5b]. Formation of a positive ion from the neutral iron compounds when their solutions are infused would seem to require that the one ferrous atom be raised to the ferric state. It is difficult to predict what the effect of this change of valence would be on complex formation, but from the appearance of the mass spectrum at low tube lens voltage with its intact three ligands, it seems that the gross structure of the original compound is retained by the compound even in its full ferric ionic state, at least for the short time required for analysis. Of course, when the iron ions are formed electrolytically they may be in the full ferric state prior to complex formation, although Blades et al. [8] found only ferrous ions formed under these conditions. In an attempt to determine the nature of any effect from such valence changes, a solution of ferrous acetate in acetic acid was examined in the same man-
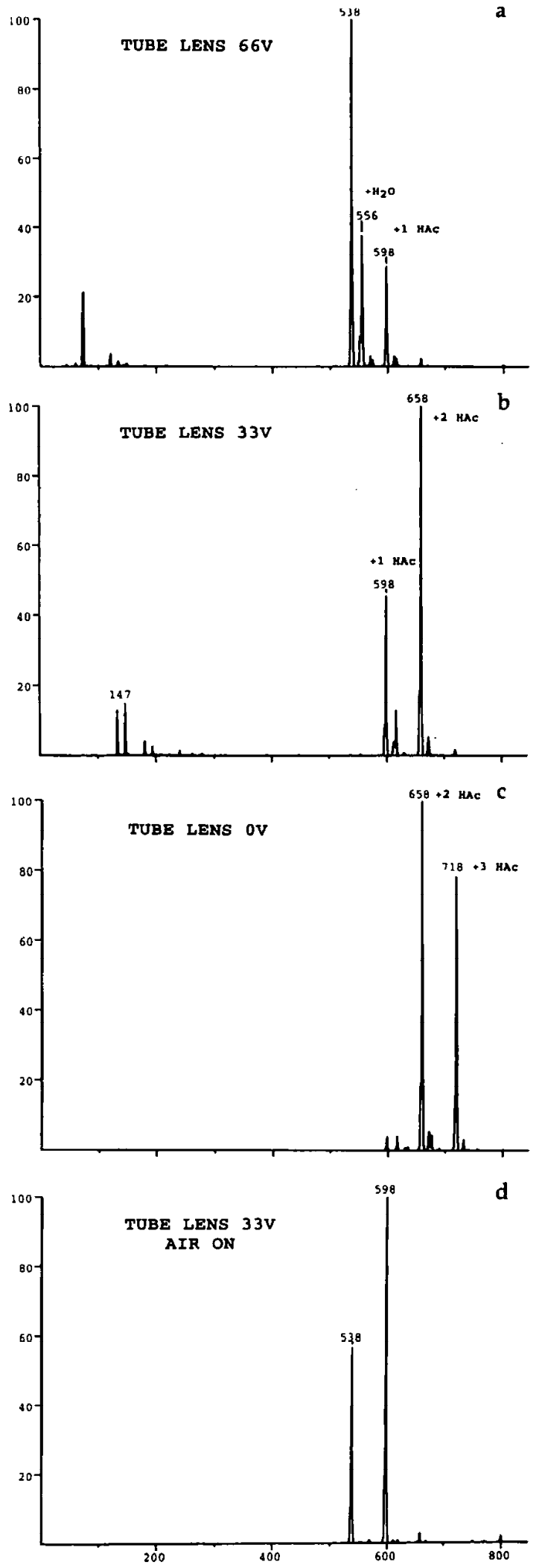

Figure 3. (a) Electrospray spectrum (Finnigan TSQ-700) of 1- $\mu \mathrm{M}$ ferric chloride in $50 \%(\mathrm{v} / \mathrm{v})$ acetic acid-methanol at $66-\mathrm{V}$ mid scan tube lens potential. (b) Same solution at 33-V tube lens potential. (c) Same solution at $0-V$ tube lens potential. (d) Same as (b) but with dry air admitted to the spray region at 20 $\mathrm{mL} / \mathrm{min}$. 
ner. In spite of the nearly colorless nature of the ferrous solution, exactly the same spectrum was obtained as with the higher oxidation state.

Returning to the evaluation of propionic acidisopropanol for the infusion electrospray of peptides, we analyzed a $50-\mu \mathrm{M}$ solution of a mixture of peptides [angiotensin I (1260 u; A1), angiotensin II (1031 u; A2), physalaemin (1266 u; P), aprotinin (6511 u; AP), and insulin $(5733 \mathrm{u} ; \mathrm{I})$ ] in $50 \%$ aqueous methanol (Figure 4 a). As with the tuning solution, $0.1 \%$ of this solution in TFA reduced the average signal (sum of all intensities) by a factor of $\sim 69$ (Figure $4 \mathrm{~b}$ versus $4 \mathrm{a}$ ). With all of the peptides except insulin the charge state also was reduced markedly and some sodium adducts were formed, possibly due to its presence in the TFA. Addition of $25 \%$ by volume of the propionic acidisopropanol solution increased the signal strongly so that now it was reduced only by an average factor of 2.3 from the original (Figure $4 \mathrm{c}$ versus $4 a$ ). Interestingly, the charge states were only partially returned to their higher value. Confirming the results of Apffel et al. [2], addition to the TFA solution of the same volume of a 1:1 mixture of methanol and acetic acid was not as effective as propionic acid-isopropanol, and the signal was still reduced by a factor of 28 from the original intensity (not shown).

Apffel et al. [2] suggested that the mechanism for improvement of the signal by propionic acidisopropanol is connected with the replacement of the TFA by the more plentiful propionic acid through mass action as the TFA preferentially evaporates from the shrinking droplet due to its greater volatility. The propionate anion with its weaker ion-pairing ability compared to the TFA anion allows easier ejection of the protonated peptide from the droplet, at least in one view of the mechanism for ion production in electrospray [9]. Success of the propionic acid-isopropanol technique with the volatile hydrochloric acid and failure of the effect to be observed with the involatile sulfuric and phosphoric acids [2] certainly supports this view. Formic acid presumably fails as a substitute for acetic or propionic acid because its stronger ionpairing ability acts in the same manner as TFA. This implies that formic acid added to a peptide should act in the same manner as TFA itself. Indeed, by using the foregoing mixture of peptides we find that the signal is first reduced by a factor of 6.8 after the solution is made $3 \%$ in formic acid and then the signal is enhanced to its original intensity by subsequent addition of propionic acid-isopropanol solution (not shown).

\section{Conclusions}

We confirm the observation of Apffel et al. [2] that the reduction in signal observed on addition of TFA or formic acid to solutions of compounds such as peptides and proteins can be partially or wholly reversed by the addition of propionic acid-isopropanol. In these
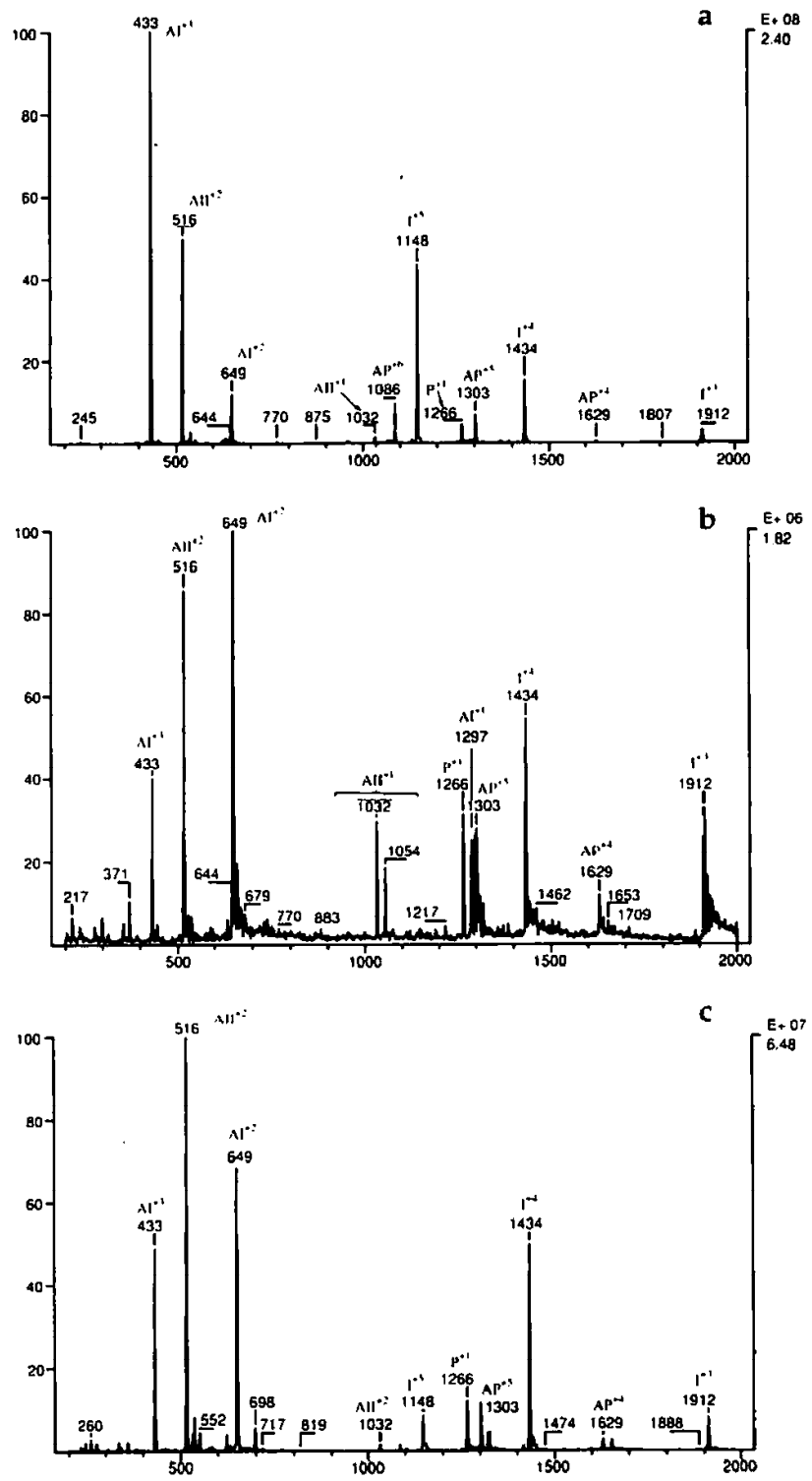

Figure 4. (a) Electrospray spectrum of a mixture of angiotensin I (A1), angiotensin II (A2), physalaemin (P), aprotinin (AP), and insulin (I) in $50 \%(\mathrm{v} / \mathrm{v})$ aqueous methanol. (b) Same solution as in (a) brought to $0.1 \%(\mathrm{v} / \mathrm{v})$ with TFA; note reduction in signal on the right of the figure. (c) same solution as in (b) with addition of $25 \%$ propionic acid-isopropanol.

studies we have identified an unusual ion with $m / z$ $622(\mathrm{~m} / \mathrm{z} 538$ with acetic acid) as a basic iron carboxylate complex and noted its tendency to attach solvent molecules. The electrospray technique clearly has considerable potential in the study of such metallic complexes.

We also have noted the presence of conventional electrolytic conditions in the Finnigan electrospray source as originally configured that may significantly affect the creation of metallic salts during electrospray and also disturbs spray stability by formation of hydrogen gas bubbles. 


\section{References}

1. (a) Hiraoka, K.; Kudaka, I. Anal. Chem. 1992, 64, 75-81; (b) Mann, M. Org. Mass Spectrom. 1990, 25, 575-587.

2. (a) Apffel, A.; Fischer, S.; Goodley, P. C.; Sahakian, J. A. In Proceedings of the 42nd ASMS Conference on Mass Spectrometry and Allied Topics; Chicago, IL, May 29-June 3, 1994; abstract 642; (b) Apffel, A.; Fischer, S.; Goldberg, G.; Goodley, P.; Kuhlmann, F. In the Proceedings of the 42nd ASMS Conference on Mass Spectrometry and Allied Topics; Chicago, IL, May 29-June 3, 1994; abstract 772. See also Hewlett-Packard Ion Notes, 1994, 9, 4-5.

3. Ijames, C. F.; Markey, S. P. J. Am. Soc. Mass Spectrom. 1994, 5, 398-406.

4. Cotten, F. A.; Wilkinson, G., Eds. Advanced Inorganic Chem- istry, 5th ed.; Wiley-Interscience: New York, 1988; pp 466, 718.

5. (a) Woehler, S. E.; Wittebort, R. J.; Oh, S. M.; Hendrickson, D. N.; Innis, D.; Strouse, C. E. I. Am. Chem. Soc. 1986, 108, 2938-2946; (b) Sumner, C. E., Jr.; Steinmetz, G. R. J. Am. Chem. Soc. 1985, 107, 6124.

6. Dziobkowski, C. T.; Wrobleski, J. T.; Brown, D. B. Inorg. Chem. 1981, 20, 679-684.

7. Gorun, S. M.; Papaefthymiou, G. C.; Frankel, R. B.; Lippard, S. J. J. Am. Chem. Soc. 1987, 109, 3337-3348.

8. Blades, A. T.; Ikonomou, M. G.; Kebarle, P. Anal. Chem. 1991, 63, 2109-2114.

9. For a recent review, see Ashton, D. S.; Beddell, C. R.; Cooper, D. J.; Green, B. N.; Oliver, R. W. A. Org. Mass Spectrom. 1993, $28,721-728$. 